CIC. Cuadernos de Información y Comunicación

ISSN: 1135-7791

http://dx.doi.org/10.5209/CIYC.60911

\title{
La innovación docente en los estudios de Comunicación: análisis documental de los proyectos financiados por las universidades públicas madrileñas
}

María Lara $^{1}$; Noelia García Castillo ${ }^{2}$; Tamara Bueno Doral ${ }^{3}$

Recibido: 15 de mayo de 2018 / Aceptado: 13 de julio de 2018

Resumen. Una educación de calidad es un pilar fundamental para el desarrollo y transformación de una sociedad. Sin embargo, la innovación docente apenas es tenida en cuenta en los programas de evaluación del profesorado que condicionan la promoción profesional del mismo. Ante este sistema que prioriza los resultados de investigación en detrimento de la enseñanza, una de las opciones que les quedan a los profesores universitarios es el diseño y participación en proyectos de innovación docente. En este artículo presentamos un análisis crítico de la situación actual en la Educación Superior, acompañado de un estudio que recoge los proyectos de innovación docente llevados a cabo en el área de Comunicación de las universidades públicas madrileñas, con el objetivo de conocer si conforman propuestas de innovación que contribuyan a las actuales demandas sociales.

Palabras clave: innovación docente, universidad pública, evaluación del profesorado, estudios de Comunicación.

\section{[en] Teaching innovation in Communication studies: documentary analysis of projects funded by public universities of the Community of Madrid}

\begin{abstract}
An education of quality is a basis for the development and transformation of society. Nevertheless, teaching innovation is barely considered by the Spanish agencies of academic staff evaluation that determine the professional promotion of lecturers. In this system that prioritizes research results to the detriment of teaching, one of the remaining options for lecturers is the design and participation in teaching innovation projects. This paper contains a critical analysis of the current situation of Higher Education, with a study that collects the teaching innovation projects in Communication studies in the public universities of the Community of Madrid, Spain, with the aim of knowing if these projects define innovation proposals that contribute to the current societal demands.

Keywords: teaching innovation, Spanish public university, academic staff evaluation, Communication studies.
\end{abstract}

Sumario. 1. Introducción: una breve reflexión en torno a los modelos educativos. 2. La innovación educativa aplicada a los estudios de comunicación. 3. Análisis de los proyectos de innovación docente en Comunicación de las universidades públicas de la Comunidad de Madrid. 3.1. Universidad de

$1 \quad$ Universidad Complutense de Madrid.

marmtez@gmail.com

2 CMI Business School, Universidad Pontificia Comillas y EAE Business School.

noeliagcastillo@gmail.com

$3 \quad$ Universidad Complutense de Madrid.

trbueno@pdi.ucm.es 
Alcalá de Henares. Convocatoria 2015/2016. Convocatoria 2016/2017. Convocatoria 2017/2018. 3.2. Universidad Carlos III. Convocatoria 2015/2016. Convocatoria 2016/2017. Convocatoria 2017/2018. 3.3. Universidad Complutense de Madrid. Convocatoria 2013. Convocatoria 2014. Convocatoria 2015. 3.4. Universidad Rey Juan Carlos. 4. Conclusiones. 5. Bibliografía.

Cómo citar: Lara, M.; García Castillo, N.; Bueno, T. (2018). La innovación docente en los estudios de Comunicación: análisis documental de los proyectos financiados por las universidades públicas madrileñas, en CIC. Cuadernos de Información y Comunicación 23, 143-156.

\section{Introducción: una breve reflexión en torno a los modelos educativos}

En los últimos tiempos, ante las deficiencias que muestra el actual sistema universitario público, son muchas las voces que se alzan reclamando una reforma educativa en la formación superior. Los estudiantes y los docentes e investigadores, experimentamos día tras día enormes dificultades provenientes de una particular visión identificada con una orientación mercantilista de la educación que se ha ido implementando en nuestro país durante los últimos años. Así se expone en la declaración de intenciones de la denominada Estrategia Universidad 2015: "La EU2015 plantea la responsabilidad académica colectiva, para que [...] se promuevan cambios de eficiencia y eficacia social y se incremente la contribución socioeconómica de la Universidad". (Ministerio de Educación, 2010:1)

Desde la fundación de las primeras universidades, como la de Constantinopla en el año 340, antecesora del concepto desarrollado posteriormente, o la de Bolonia (Italia), que recibió el título de Universidad en 1317, hasta nuestros días, las instituciones de educación superior han experimentado una evolución dispar según los modelos educativos imperantes en cada país, pero siempre acorde a las concepciones propias de las sociedades en las que se circunscriben. En la actualidad, las universidades se ven influenciadas por el pensamiento capitalista dominante en este mundo globalizado y también, por sus correspondientes modelos políticos. No es de extrañar por tanto, que los criterios de eficiencia cuantitativa sean los que se evalúen de manera preferente en cuanto a los méritos alcanzados en investigación, en claro detrimento de la innovación educativa, por poner un ejemplo. En este punto, resulta relevante plantearnos la siguiente cuestión: ¿pueden dedicar mucho tiempo y trabajo a la innovación docente los profesores e investigadores que quieran rápidamente progresar en la carrera académica, cuando es un criterio minusvalorado en las evaluaciones que les van a permitir ascender?

En cuanto al modelo educativo que impera actualmente en las universidades, podemos decir que está fundamentalmente centrado en la profesionalización. Sin duda que una de las funciones principales de la institución universitaria es formar de modo especializado a los futuros profesionales, éstos han de poder resolver las exigencias que se les van a requerir en el mercado laboral, pero existen también otros valores educativos que no deberían ser olvidados: el desarrollo del pensamiento más allá de todo dogma, la transmisión de la cultura, el perfeccionamiento ético, la transformación de la sociedad, el desarrollo integral del ser humano, la formulación de nuevas soluciones adaptativas a cada momento histórico, entre otros. Las decisiones que se toman en el ámbito educativo tienen una consecuencia directa en nuestra construcción social y pueden favorecer grandes posibilidades de cambio y de superación de antiguos paradigmas. No obstante, también pueden producir inmovilismos 
según la orientación que se le otorguen a esas políticas, pero siempre supone un ámbito en el que se refleja una mentalidad social comúnmente aceptada. Por ejemplo, que el crecimiento de una nación se mida casi exclusivamente en términos monetarios es un claro indicador de los valores sociales mayoritarios.

Con relación al significado e importancia de la educación en una sociedad, el genial filósofo Ortega y Gasset manifiesta:

Si educación es transformación de una realidad en el sentido de cierta idea mejor que poseemos y la educación no ha de ser sino social, tendremos que la pedagogía es la ciencia de transformar sus sociedades. Antes llamamos a esto política: he aquí, pues, que la política se ha hecho para nosotros pedagogía social y el problema español es un problema pedagógico. (Ortega, 1946).

Así, para Ortega la urgente necesidad de reformar la Universidad depende en primer lugar de crear "usos nuevos" y para ello, resulta esencial determinar cuál es su misión, el propósito desde el que orientar esos cambios (Ortega, 1998). Además, la educación tiene como foco principal al ser humano y su relación con el ámbito social mayor, siendo esta transformación de la realidad posible cuando se realiza en base a un "ideal". Identifica así a la educación con la transformación social (Ortega, 1946).

También decía el filósofo que debía concebirse una formación que preparara a los seres humanos para la movilidad, ya que la inercia natural del entorno social ha tendido siempre a la inestabilidad (Daros, 2017: 2). Estas afirmaciones toman gran relevancia en este momento histórico en el que el crecimiento exponencial científico y tecnológico nos brinda múltiples oportunidades de desarrollo. Así, debido a la aceleración de cambios a la que nos enfrentamos, se hace necesario el estudio de conocimientos integrales que ayuden a la persona a adaptarse a un entorno en permanente transformación y a generar un criterio propio. En este sentido, los nuevos métodos docentes deberían hacer hincapié en el aprendizaje de aptitudes, la innovación docente, las metodologías colaborativas, etc., pero también avanzar hacia un conocimiento transdisciplinar, capaz de abordar de forma conjunta y desde múltiples enfoques la complejidad de las problemáticas actuales.

\section{La innovación educativa aplicada a los estudios de comunicación}

Específicamente, en el caso de las carreras de Comunicación, la universidad ostenta una creciente responsabilidad, ya que como advierte Pedro-Carañana (2016), este campo interviene en "el funcionamiento y cambio de las sociedades [...] y los sistemas sociales influyen sobre la comunicación". En nuestro campo de especialización, puesto que los públicos están expuestos a innumerables estímulos desde múltiples canales de información, la alfabetización mediática se ha convertido en una de las enseñanzas vitales para el ciudadano y no sólo para los profesionales de la comunicación. Según Lee (2013), ante la saturación de datos que puede conllevar la evolución de las TIC, deben formarse individuos con autonomía de pensamiento. Así podrían distinguir aquellos contenidos con cierta veracidad y relevancia frente a las abundantes fake news que circulan por la red. Los alumnos habrán de aprender a buscar la información, seleccionar los contenidos relevantes, organizarlos y compar- 
tirlos, para lograr que la población esté "equipada con altos niveles de reflexión y espíritu crítico" (Lee, 2013). El analfabeto no hará referencia al individuo que no sepa leer, sino a aquel que no conozca cómo aprender (Lee, 2013). Como determinó la National Communication Association (Morreale et al., 2000) un criterio más elaborado mejoraría además las relaciones personales, el entendimiento intercultural, permitiría el empoderamiento de todos los miembros de la sociedad y favorecería la participación en la vida pública.

La llamada "alfabetización" ha sido renombrada por numerosos teóricos para actualizar el concepto tras el resurgimiento de las nuevas tecnologías. De acuerdo a Mackey y Jacobson (2011), la "metalfabetización” contribuye a la implicación en el mundo digital desde el juicio meditado y el trabajo colaborativo. Taylor utiliza "transalfabetización" para definir la interacción a través de diferentes plataformas y herramientas con el fin de lograr un entendimiento más avanzado (Todd, 2000). El perfeccionamiento de las destrezas comunicativas serviría para poder discernir entre los diferentes medios, interpretar en qué condiciones son producidas las noticias o creaciones audiovisuales que se difunden e intervenir en el espacio público mediante el intercambio de opiniones. Para los alumnos de Comunicación resulta aún más relevante adquirir esta capacidad ya que según apunta María Acaso para convertirse en productor de contenidos antes hay que ejercer como consumidor crítico (Blázquez, 2017).

Otras habilidades que se hacen imprescindibles para desenvolverse en la vida diaria y para prosperar en el entorno laboral serían la capacidad para resolver los problemas complejos y el fomento de la creatividad. Para lograr todas estas aptitudes reseñadas e integrarlas de forma adecuada, se precisa una modificación de metodología docente que introduzca estos usos en el aula como una "competencia transdisciplinar" presente en todas las áreas y al tiempo, una "transformación en las prácticas y el rol del profesorado" (Lau, 2013).

Diversas propuestas sugieren personalizar la formación creando una experiencia diferente en cada alumno, fomentando también la participación y el rendimiento conseguido, reemplazando antiguas pautas "por nuevos modelos más centrados en el estudiante" (McLoughlin y Lee, 2008). Ante una mayor capacidad de los alumnos para aprender de forma autónoma, se reduciría su dependencia del docente tradicional, que de acuerdo a Woods (citado en Scott, 2015: 7) dejaría de ser la fuente principal de conocimiento y se transformaría en "mentor y mediador del proceso de aprendizaje". Ejercería como motivador de sus estudiantes, para que buscaran aquello que les despertara mayor interés (Malone y Smith, citados en Meyer et al., 2008). De acuerdo a Gilbert (2005), en lugar de centrarse en la transmisión de contenidos, el educador les ayudaría a perfeccionar sus habilidades, a colaborar en grupos reducidos y a entender cada asignatura como un sistema de pensamiento "con sus propios códigos, métodos, ventajas y limitaciones". En esta nueva estructura, todos los miembros de la clase se harían responsables del aprendizaje de sus compañeros (Srinivas, citado en Laal et al., 2012). Además, desde esta metodología docente, no se contempla al alumno como un ser pasivo, sino que se busca "despertar" su intención.

Numerosas propuestas innovadoras en educación toman de la neurociencia la premisa de que el cerebro "necesita emocionarse para aprender", aunque doctores como Francisco Mora, piden prudencia ya que en este campo aún quedan múltiples dudas por resolver. Añade que "Es necesario despertar la curiosidad, que es el mecanismo cerebral capaz de detectar lo diferente en la monotonía diaria. Se presta aten- 
ción a aquello que sobresale. [...] Las emociones sirven para almacenar y recordar de una forma más efectiva." (Torres, 2017)

Multitud de iniciativas están surgiendo en todo el planeta, expresándose a través de estudios fundamentados, teóricos de prestigio y también a partir de maestros anónimos que comparten sus experiencias personales y sugerencias a la comunidad educativa. Todo ello es un reflejo de la aspiración generalizada que busca un gran cambio en la concepción de la enseñanza, un cambio que como decía Unamuno, habría que llevarlo a cabo primero "en las cabezas de los que enseñan, o por lo menos, en las de los que han de enseñar." (Bastons, 2000: 45).

Como docentes no somos ajenos a estas necesidades y, en menor o mayor medida, tratamos de estar al corriente de las metodologías que nos resultan más afines. Sin embargo, a pesar de las buenas intenciones de muchos, la realidad en el entorno universitario impone sus limitaciones de todo tipo: desde la falta de recursos, a la masificación en las aulas y la imposibilidad de responder a todas las exigencias que se le piden hoy en día a la figura del profesor.

A pesar de estas dificultades, el papel de las universidades públicas sigue siendo fundamental en el avance de la sociedad democrática y requiere adaptarse a las circunstancias. Ya en la Edad Media, existía una preocupación por dotar de conocimientos pragmáticos las enseñanzas universitarias. En la actualidad han sido varias las instituciones nacionales que han modernizado sus planes de estudio para adecuarlos a las tendencias más novedosas del mercado laboral. En el caso concreto de las carreras de Comunicación, los recién licenciados deberían haber desarrollado durante su formación las destrezas ya reseñadas a lo largo del artículo y también, dominar los aspectos técnicos y narrativos necesarios para generar contenidos audiovisuales y escritos, adaptándolos al estilo requerido y a un determinado formato. Esto implica conocer las claves del lenguaje de la comunicación, así como los recursos tecnológicos para elaborar los diversos materiales según el medio y el canal: noticias, fotografías, blogs, páginas web, programas de radio, guiones, documentales, vídeo-arte, vídeos institucionales, spots publicitarios, series de TV, películas, entre otros. A pesar de que a los estudiantes actuales se les presupone ser "nativos digitales", según recuerda Fouce acerca de los alumnos de periodismo: "no todos están familiarizados con las herramientas necesarias para crear un blog: manejo de las interfaces, edición de fotografía y vídeo, generación de enlaces...”. (2014: 3)

Los profesores especializados en muchas de estas áreas han de mantenerse al día de los últimos avances ya que la enseñanza de dichas disciplinas exige un alto dominio técnico y en ocasiones, artístico, que los estudiantes han de ejercitar a través de sesiones prácticas. El carácter de estas clases hace necesario estar familiarizado con el mundo profesional, lo que únicamente se consigue mediante la experiencia de participar en la creación de un proyecto real. A diferencia de otras carreras en las que se evalúa positivamente ser una autoridad en la práctica, como ocurre con estudios como Medicina, para impartir las asignaturas de "Dirección Cinematográfica", "Realización Publicitaria", "Edición y Postproducción" o "Fotoperiodismo", no se reconoce apenas el mérito de creación de una obra gráfica o audiovisual. Cuando se desarrolla un doble perfil, de docente y profesional, al participar en un proyecto de estas características, se llevan a cabo labores de investigación que validan los conceptos para luego poder elaborarlos y transmitírselos al alumnado. En cambio, en la actualidad resultan casi siempre más valorados los textos escritos, incluso aquellos que no ahondan en un conocimiento profundo de la obra a la que hacen referencia. 
Puede resultar extremadamente complejo establecer sistemas de evaluación que se ajusten a todos los perfiles, ya que según la carrera y también de acuerdo a la materia impartida, variarán los requisitos indispensables en el docente, en relación con sus líneas principales de investigación, trayectoria académica, habilidad para comunicarse con el estudiante, capacidad de adaptación... Por este motivo, resultaría interesante una futura revisión de la especificidad de cada materia, como apuntaba el catedrático emérito Juan Manuel de Faramiñan: "contemplar las diferencias de las distintas carreras y titulaciones a la hora de establecer el control de calidad." (El camino a la investigación universitaria, 2017).

Como señalábamos a lo largo del artículo, en la docencia universitaria, al margen de la propia especialidad, resultaría primordial reflexionar sobre la labor educativa y la metodología más adecuada que aplicar en cada ámbito, haciendo un ejercicio de autocrítica que no siempre resulta sencillo. Una oportunidad para ello son los Proyectos de Innovación Docente, puesto que muchos de ellos estimulan buenas prácticas, plantean una experimentación con las herramientas de aprendizaje, presentan diferentes recursos educativos, y en ocasiones, se adaptan al desarrollo de las nuevas tecnologías, como es el caso de la formación online.

\section{Análisis de los proyectos de innovación docente en Comunicación de las universidades públicas de la Comunidad de Madrid}

Para la realización de este apartado del estudio se ha recurrido a la información disponible en las páginas web de las distintas instituciones educativas, principalmente a las resoluciones de las respectivas convocatorias referentes a la Innovación Docente. El objetivo principal de este trabajo consiste en observar las tendencias que están adoptando los profesionales de la Educación Superior para la enseñanza de la Comunicación, así como estudiar si sus líneas de mejora comprenden aquellos aspectos anteriormente mencionados como necesarios para un avance en el modelo educativo de la universidad pública española.

A continuación, mostramos los resultados tras el análisis documental aplicado a la información disponible de las cuatro universidades públicas de la Comunidad de Madrid en las que entre su oferta formativa se encuentra, al menos, un estudio de grado en el área de Comunicación. Para la selección de los distintos proyectos y premios se ha tenido en cuenta que los docentes participantes pertenezcan a departamentos de Comunicación o que ejerzan su docencia en grados de Comunicación.

\subsection{Universidad de Alcalá de Henares}

Como anuncia en su página corporativa ${ }^{4}$, la institución mantiene un Programa de Innovación Docente que, desde 2010, ha desarrollado líneas estratégicas relacionadas con Grupos de Innovación Docente, convocatorias de Proyectos y Encuentros de Innovación Docente Universitaria. Estas iniciativas comparten unos puntos comunes basados en la interdisciplinariedad, el aprendizaje activo del alumnado y la evaluación continua. Como metodologías para la innovación docente, las convocatorias mencionan explícitamente el aprendizaje basado en problemas (conocido por PBL

4 https://www3.uah.es/ice/ID/ 
por sus siglas en inglés) y el aprendizaje reflexivo, así como el recurso de las nuevas tecnologías. Por último, la Universidad de Alcalá de Henares procura dotar a su profesorado de las habilidades y competencias necesarias para la consecución de tales fines por medio del Plan de Formación del Profesorado.

Proseguimos nuestro estudio con la relación de proyectos aprobados, con o sin financiación, en la "Convocatoria de Proyectos para el fomento de la Innovación en el proceso de Enseñanza-Aprendizaje”. Al poder encontrar la información necesaria en las respectivas resoluciones definitivas, aportamos la información relativa a las convocatorias 2015/2016, 2016/2017 y 2017/2018:

\section{Convocatoria 2015/2016}

- "La imagen como herramienta docente en la universidad", Julián de la Fuente Prieto.

- "La Revista Digital una actividad complementaria para la formación del alumnado en Com. Audiovisual”, Rut Martínez Borda.

\section{Convocatoria 2016/2017}

- "La comunicación en los procesos de formación. Creación de contenidos audiovisuales en contextos interactivos", Sara Cortés Gómez.

\section{Convocatoria 2017/2018}

- "La comunicación como elemento interdisciplinar: creación y difusión de contenidos multimedia”, María Ruth García Pernía.

Tras un exhaustivo proceso de búsqueda, no ha sido posible encontrar información referente a los objetivos o resultados de estos proyectos. La única información que hemos podido localizar en la web corporativa es que todos los coordinadores de los citados proyectos pertenecen al Grupo de Investigación "Imágenes, palabras e ideas" entre cuyas líneas de investigación encontramos: "Diseñar escenarios educativos innovadores" y buscar "relaciones entre contextos educativos formales y no formales", el uso de "instrumentos culturales y nuevas alfabetizaciones" como es el caso de "los videojuegos" y la "utilización de recursos audiovisuales e informáticos" en las "metodologías visuales de investigación".

Por su parte, Julián de la Fuente Prieto es integrante del Grupo de Innovación Docente "La imagen como herramienta docente en la universidad"6, entre cuyas líneas y temáticas de investigación contempla: la docencia en Arquitectura, la docencia en Comunicación Audiovisual, la realidad aumentada como herramienta docente, la evaluación en estudios universitarios, nuevas formas de docencia y los Trabajo Fin de Grado y de Máster junto a la práctica profesional como herramienta docente.

Observamos así de qué manera se siguen algunos de los principios fomentados desde la institución como son la innovación docente interdisciplinar y el recurso a las nuevas tecnologías aplicables al entorno de enseñanza, como lo son los video-

https://www.uah.es/es/investigacion/unidades-de-investigacion/grupos-de-investigacion/ https://www3.uah.es/ice/ID/documentos/GGI_plantilla.pdf 
juegos, las revistas digitales, los contextos interactivos multimedia y la realidad virtual.

Posteriormente se observará que el número de proyectos de innovación docente concedidos por otras instituciones como la Universidad Carlos III o la Universidad Complutense son mayores. Tales resultados son comprensibles ya que la Universidad de Alcalá de Henares cuenta con una única titulación del área de Comunicación: el Grado en Comunicación Audiovisual.

\subsection{Universidad Carlos III}

Como apunta la propia institución en su decimoquinta convocatoria de Apoyo a Experiencias de Innovación Docente", la innovación docente es "parte de la estrategia de la universidad". Para ello considera que las nuevas tecnologías son de vital importancia al permitir "repensar los métodos y las herramientas educativas" de manera que éstos acentúen su "carácter digital, social y colaborativo" a la vez que se promueven "los formatos híbridos" y se fomentan las "experiencias educativas enriquecedoras". Ejemplo de lo anterior son los MOOCs (Massive Open Online Courses), gracias a los cuales esta universidad presume de ser una de las pioneras al adoptar dicho formato a la docencia reglada. En este contexto, la mencionada convocatoria pretende potenciar las características de la Educación Digital para la Innovación educativa.

Al igual que en el caso de la Universidad de Alcalá, ha sido posible encontrar la información necesaria en las resoluciones definitivas de las tres últimas convocatorias. No obstante, también coincide la falta de información respecto a los objetivos y los resultados de cada uno de los proyectos concedidos.

\section{Convocatoria 2015/2016}

- "La formación en investigación de los estudiantes de Periodismo y Comunicación Audiovisual. Nuevas estrategias para la participación en red y semillero de ideas para la innovación en el campo", Matilde Eiroa San Francisco.

- "Blog bilingüe para la adaptación de metodologías de docencia y aprendizaje de la asignatura de Psicología de los Medios / Media Psychology al actual entorno digital", Sonia García López.

Convocatoria 2016/2017

- "La producción visual como proceso intelectual y creativo. La bitácora como testimonio y como instrumento de evaluación", Alejandra Walzer Moskovic.

- "Tecnologías para la producción audiovisual. Curso básico", Francisco Utray Delgado.

- "Periodismo de las dos orillas", Miguel Ángel del Arco Bravo.

Convocatoria 2017/2018

- "Pensamiento complejo en Información y Comunicación: aprendizaje a través de la participación en redes sociales", Mercedes Caridad Sebastián. 
- "Distintas caras de la noticia: radio, web y televisión", Eva Herrero Curiel.

- "El reportaje periodístico, una herramienta para la comprensión de la historia contemporánea”, Carlos Rodríguez López-Brea.

Los principales objetivos de la última convocatoria eran propiciar y extrapolar a otras asignaturas y titulaciones "las buenas prácticas docentes y la mejora de recursos didácticos y educativos", fomentar la participación activa e implicación del alumnado y experimentar con nuevas formas de evaluación.

No obstante, los propios convocantes han reconocido la dificultad de premiar aquellas propuestas que "no suponían un gran cambio innovador, pero que sí mejoraban algún aspecto de lo que se estaba haciendo hasta el momento". Para los organizadores, no conceder estas iniciativas, como incentivar la participación -el debate o la generación de nuevos contenidos- "daba una imagen equivocada de lo que se pretende fomentar", mientras que su concesión podía suponer un agravio comparativo con aquellos proyectos que fueran realmente innovadores e implicaran un verdadero esfuerzo. Para poner fin a esta disyuntiva, decidieron dividir la convocatoria 2017/2018 en dos modalidades: las de tipo A que "introduzcan una innovación de mayor envergadura" y las de tipo B "que mejoren la práctica docente existente en una asignatura concreta, pero que no supongan una innovación sustancial en términos absolutos".

En el caso concreto del área de Comunicación, todas las propuestas aprobadas corresponden a la modalidad B; y en términos generales, el número de concesiones en las tres últimas convocatorias tiende a permanecer estable. Siguiendo con las líneas principales priorizadas por la entidad en lo que a innovación docente se refiere, varios proyectos recurren a ciertos usos de las nuevas tecnologías, como son las redes sociales y los blogs, que forman parte de la realidad cotidiana del alumnado actual. Ello con el objetivo de fomentar la interacción, la participación y el razonamiento crítico o "pensamiento complejo", necesarios en cualquier asignatura, pero todavía más en la "formación en investigación". Estas "nuevas formas para la participación de los alumnos" resultan especialmente valiosas en el caso de asignaturas con opción bilingüe, en la que los participantes pueden mostrar reticencias a intervenir por miedo a su falta de dominio del idioma. Cabe mencionar igualmente aquellas "nuevas formas de evaluar" que fomentan una evaluación continua necesaria para converger con el Espacio Europeo de Educación Superior.

\subsection{Universidad Complutense de Madrid}

A partir de 2016 la institución no revela los títulos de los proyectos en las resoluciones de concesión, sólo las referencias numéricas referentes al orden de solicitud. Es por este motivo que se ha recurrido para la presente investigación a las resoluciones de las convocatorias de los años 2013, 2014 y 2015, en las que figura el título del proyecto, pero no el docente coordinador del mismo.

En el caso de la convocatoria de Proyectos de Innovación y Mejora de la Calidad Docente (PIMCD) $2015^{8}$, se priorizan una serie de estrategias que, en líneas generales, coinciden con las de las ediciones anteriores correspondientes a 2013 y 2014 : fomentar la participación del alumnado, potenciar el emprendimiento, adquisición y

https://www.ucm.es/PIMCD-2015 
evaluación de competencias genéricas y transversales, docencia en inglés, enseñanza virtual, difusión de las tendencias del Acceso abierto (Open Access) en la investigación y docencia, redes sociales en el aprendizaje, el aprendizaje-servicio (ApS), uso de entornos colaborativos, recursos educativos en abierto (Open Educational Resources) y usos complementarios del Campus Virtual.

En la resolución de la convocatoria de 2013 solamente se publican los títulos de los proyectos y las facultades a las que pertenecen. Desde la convocatoria de 2014 se produce un cambio en la política de visibilización de los resultados de los proyectos de innovación docente y se publican a través de la plataforma en abierto E-Prints Complutense.Gracias a dichos informes, es mayor la información que aportamos sobre algunos proyectos de la convocatoria de 2014 y la totalidad de la de 2015 . Tras aplicar los criterios de búsqueda pertinentes en el navegador de la plataforma de acceso abierto, desconocemos la causa de la publicación parcial de resultados para la convocatoria de 2014.

\section{Convocatoria 2013}

- "Nuevos retos y sostenibilidad del Método docente de proyecto social real".

- "La Huella Digital. Revista Universitaria".

- "Uso de dispositivos inteligentes (tablets, smartphones...) como herramientas educativas en el campus virtual y portales educativos (Publidocnet): aplicaciones prácticas en la asignatura Documentación Informativa”.

\section{Convocatoria 2014}

- "Nuevos modelos y aplicaciones transversales del método docente de proyecto social/cultural real", Liisa Hänninen. Aprendizaje activo con entidades del tercer sector y fomento del espíritu emprendedor.

- "Massive Open Online Course de narrativa audiovisual para campus virtual: el alumnado como realizador de materiales didácticos innovadores".

- "Qué necesitan los estudiantes de Periodismo para su inserción laboral. Análisis de la demanda del mercado laboral de las empresas de comunicación", Fernando Peinado Miguel. Estudio de las ofertas de prácticas en el sector ofrecidas a través del portal de empleo de la universidad..

- "Semiótica, tecnología digital y práctica periodística", Héctor Fouce. Aprendizaje reflexivo y activo sobre la complejidad de la comunicación mediante las tecnologías 2.0. y la práctica profesional.

- “Observatorio de los Informativos de Televisión. Análisis de contenidos en la "nube'. Aprendizaje colaborativo e interactivo".

\section{Convocatoria 2015}

- "Glosario social multimedia de innovación educomunicativa y TIC", Isidro Moreno Sánchez. Entre sus objetivos se encontraba potenciar herramientas virtuales que, en un ámbito internacional e interdisciplinar, ayudasen a los docentes en el desempeño de su labor.

- "Uso de redes creativas y colaborativas en las asignaturas de grado de Comunicación y Sociología Política con perspectiva de Género", Eva Aladro 
Vico. Buscaba mejorar el sistema de enseñanza-aprendizaje, centrado en la búsqueda e integración de información, mediante el uso de redes sociales y creativas.

- "Realización y difusión de un Massive Open Online Course de narrativa audiovisual avanzada: el alumnado como creador de recursos educativos abiertos", María Luisa García Guardia. Facilitar la interacción didáctica del profesorado y el alumnado a través de los MOOCs.

- "Taller y festival de spots publicitarios de las Asignaturas y los TFG vinculados al área de Producción y realización publicitaria”, Juan García Crego. Pretendía motivar a los alumnos y dotarles de competencias profesionales y de un aprendizaje autónomo.

- "Innovación docente y desarrollo de proyectos profesionales a través de la cooperación y la inteligencia colectiva", Laia Falcón Díaz-Aguado. Desarrollo de competencias con las que el alumno de los últimos cursos pueda diseñar un proyecto propio con el que afrontar su vida profesional.

- "Sombras de Luna. Ampliación del uso de este blog especializado para aplicarlo a la docencia de asignaturas impartidas en Historia del Arte (Facultad de Geografía e Historia) y Estética y Semiótica (Facultad de Filosofía) y Facultad de CC. INF.", Luis Deltell. Este proyecto interfacultativo fomentó el uso de fondos fílmicos y la divulgación del debate científico sobre la Comunicación Audiovisual entre el alumnado.

- "Observatorio de los Informativos de Televisión. Análisis de contenidos en la "nube'. Aprendizaje colaborativo e interactivo", Rafael Díaz Arias. Integración de los alumnos en un análisis de los contenidos de las principales cadenas generalistas españolas.

- "Periodismo y semiótica: tecnologías digitales y prácticas informativas", Héctor Fouce. Partió de la producción colaborativa y utilizó nuevos modelos de evaluación basados en la práctica profesional en el contexto de la asignatura "Semiótica de la comunicación de masas".

- "Metodologías docentes innovadoras en la enseñanza de posgrado en Comunicación Audiovisual y Publicidad: recursos, técnicas y prácticas en la enseñanza virtual y presencial". Francisco A. Zurian. Proyecto interdepartamental dirigido a desarrollar distintas actividades tanto presenciales como virtuales en torno a la investigación de la Comunicación.

Puede observarse cómo los proyectos de innovación docente concedidos en el área de Comunicación aumentan considerablemente en cada convocatoria. Algunos de estos proyectos tienen continuidad en distintos cursos, lo que demuestra al menos cierto grado de éxito y sostenibilidad. La apuesta por proyectos interdepartamentales e incluso interfacultativos favorece también la interdisciplinariedad y la difusión de metodologías docentes innovadoras. Por último, la publicación en abierto de un informe de resultados de cada proyecto fomenta la trasparencia de la institución y visibiliza la labor de los docentes.

\subsection{Universidad Rey Juan Carlos}

En esta institución el esfuerzo por fomentar la innovación docente se concentra en la enseñanza a distancia, ello se observa no sólo en la denominación, sino en los obje- 
tivos del "Centro de Innovación en Educación Digital: URJC online": apoyo a la docencia reglada a través del Aula Virtual, soporte técnico en el entorno virtual, formación técnico-pedagógica de docentes, innovación pedagógica mediante proyectos de tecnología educativa, diseño y producción de materiales para la enseñanza semipresencial y a distancia“... y desarrollo de proyectos de investigación en el ámbito de las Tecnologías de la Información y la Comunicación aplicadas al ámbito educativo, y, concretamente, aquellas relacionadas con las modalidades semipresencial o a distancia".

En este marco de actividad, la Universidad Rey Juan Carlos convoca anualmente el "Premio Profesores Innovadores" para "mejorar la impartición de la docencia en todos sus ámbitos mediante la incorporación de las TIC a los procesos de enseñanza y aprendizaje así como al desarrollo de prácticas educativas abiertas y metodologías activas" $"$.

En su IV convocatoria correspondiente a 2017, uno de los premiados fue el profesor Manuel Gértrudix Barrio por el proyecto "Desarrollo de competencias profesionales en periodismo semipresencial mediante REAs, contrato de tareas y Plan Keller" como mejor práctica educativa de aplicación de herramientas o servicios TIC en Grados semipresenciales.

En la III edición celebrada en 2016, el premio a la primera y segunda mejor práctica educativa innovadora recayó sobre profesoras del área de Comunicación Audiovisual. Belén Puebla Martínez con el proyecto "Aula de literatura: blogs, aplicaciones didácticas para dispositivos y juegos interactivos desde el trabajo colaborativo", y Gema Alcolea Díaz con la iniciativa "El proceso de enseñanza-aprendizaje a través del juego en la Universidad”. En cambio, ningún docente de Comunicación resultó premiado en la segunda convocatoria de 2015.

Uno de los objetivos de los premios "Profesores Innovadores" es también generar un banco de buenas prácticas docentes de acceso público a través de su web corporativa, por lo que, al igual que la Universidad Complutense de Madrid, esta institución aporta transparencia y visibiliza la labor docente. No obstante, no ha sido posible acceder a la información detallada de las propuestas ganadoras, por lo que es posible que, tal y como se indica en la convocatoria, la divulgación se limite al "conjunto de docentes de la universidad". Para concluir, las propuestas premiadas se restringen a la educación a distancia; de modo que desconocemos las iniciativas que se emprenden de manera desinteresada por los docentes en Comunicación de los grados presenciales.

\section{Conclusiones}

Tanto en las políticas aplicadas en el marco del plan de reforma de las universidades en España (EU2015) durante los últimos años, como en los sistemas de evaluación del profesorado (ANECA), se priorizan sustancialmente los méritos investigadores que aporta el docente, en claro detrimento de su labor educativa.

\footnotetext{
9 https://www.urjc.es/universidad/institutos-y-centros/1906-centro-de-innovacion-en-educacion-digital-urjconline\#actividades-en-el-centro-de-innovacion-en-educacion-digital-urjc-online

$10 \mathrm{https}: / /$ online.urjc.es/es/pi
} 
Como consecuencia de un sistema de formación superior que promueve primordialmente la rentabilidad económica, se han minusvalorado importantes valores educativos. Entre otros, los implícitos en el desarrollo de un pensamiento crítico, la dimensión ética, el planteamiento de soluciones innovadoras y adaptativas a las sociedades en continua transformación, el cuestionamiento de las creencias que se tienen acerca de la realidad circundante y el aprendizaje de una visión más amplia que, desde múltiples enfoques, aborde la complejidad de las problemáticas actuales.

Una oportunidad para mejorar el actual sistema educativo, la encuentran los profesores en los Proyectos de Innovación Docente, puesto que muchos de ellos promueven el estudio de conocimientos integrales que ayudan a la persona a adaptarse a un entorno en permanente transformación. Algunos aspectos a destacar en aquellos proyectos elaborados en los estudios de comunicación son: el aprendizaje de aptitudes, la innovación docente, el desarrollo de un pensamiento crítico, las metodologías colaborativas, la implementación de las nuevas tecnologías y los cambios en la tradicional relación alumno-profesor, entre otros.

Tras el análisis documental efectuado, concluimos que en los proyectos de innovación docente concedidos en el área de Comunicación por las universidades públicas de la Comunidad de Madrid se recogen algunos de los valores necesarios para la formación de profesionales y ciudadanos del siglo XXI, contribuyendo así en parte a las demandas sociales de la actualidad. Se conjugan las posibilidades que ofrecen ciertos productos de las nuevas tecnologías con el desarrollo en el alumnado de una adquisición de conocimientos autónoma y reflexiva, un espíritu crítico y un trabajo colaborativo e interactivo. A su vez, muchas iniciativas pretenden mejorar la inserción laboral del alumnado, adecuando los contenidos a la práctica profesional real y considerando nuevos modos de evaluación que complementan el progreso intelectual con el proceso creativo. Además, se favorece la interdisciplinariedad necesaria para alcanzar los nuevos modelos de alfabetización, propios de la revolución digital de la información que estamos viviendo.

No obstante, sería necesario que las universidades compartieran en acceso abierto el resultado de sus proyectos de innovación docente, para que otros investigadores y docentes pudieran avanzar en las distintas líneas posibles de una forma realmente innovadora, como exige el método y la colaboración científica.

\section{Bibliografía}

Bastons, C. (2000). "Calas a la enseñanza española a fin de siglo: Unamuno, tradición y modernidad“. En C. Flórez (Coord.). Tu mano es mi destino. Actas del Congreso internacional Miguel de Unamuno. Salamanca: Universidad de Salamanca, p. 45.

Blázquez, L. (2017). "Los exámenes no sirven de nada". El Mundo, 9 Octubre. Disponible en: http://www.elmundo.es/papel/cultura/2017/10/09/59d61827e2704e704b8b4651.html

Fernández, R. (2018). "La Universidad, cuestión de Estado". El País, 27 Abril.

Fouce, H. (2014). Semiótica, tecnología digital y práctica periodística. Proyecto de Innovación y Mejora de la Calidad Docente UCM, p. 3. Disponible en: http://eprints.ucm. es/36458/1/2016\%20Memoria\%20PIMCD\%20Semi\%C3\%B3tica.pdf

Gilbert, J. (2005). Catching the Knowledge Wave? The Knowledge Society and the Future of Education. Wellington, Nueva Zelanda: NZCER Press. 
Lau, J. (2013). “Conceptual Relationship of Information Literacy and Media Literacy”. En A. Lee, J. Lau, T. Carbo y N. Gendina, Conceptual Relationship of Information Literacy and Media Literacy in Knowledge Societies. París. Francia: UNESCO, pp. 3-75.

Lee, A. (2013). "Literacy and Competencies Required to Participate in Knowledge Societies". En A. Lee, J. Lau, T. Carbo y N. Gendina, Conceptual Relationship of Information Literacy and Media Literacy in Knowledge Societies. París. Francia: UNESCO.

Mackey, T. P. y Jacobson, T. E. (2011). "Reframing information literacy as a metaliteracy". College and Research Libraries, 72(1), pp. 62-78.

McLoughlin, C. y Lee, M.J.W. (2008). “The three p's of pedagogy for the networked society: personalization, participation, and productivity. International Journal of Teaching and Learning in Higher Education, 20(1), pp. 10- 27.

Meyer, B., Haywood, N., Sachdev, D. y Faraday, S. (2008). Independent Learning: Literature Review. Nottingham. Reino Unido: Department for Children, Schools and Families.

Ministerio de Educación (2010). Estrategia Universidad 2015. Contribución de las universidades al progreso socioeconómico español. Madrid: Secretaría general técnica. Catálogo de publicaciones del Ministerio. Disponible en: http://www. mecd.gob.es/docroot/universidad2015/flash/eu2015-flash/document.pdf

Morreale, S.P., Osborn, M.M. y Pearson, J.C. (2000). "Why Communication is Important: A Rationale for the Centrality of the Study of Communication", Journal of the Association for Communication Administration, 29, pp. 1-25.

Ortega y Gasset, J. (1946). La pedagogía social como programa político. Obras completas. Madrid: Occidente, I, pp. 494-513. Disponible en: http://www.madrid. org/cs/Satellite?b lobcol=urldata\&blobheader=application $\% 2$ Fpdf\&blobheaderne $1=$ Content-Disposition \&blobheadervalue1=filename\%3Dparticipacion_08.pdf\&bl obkey=id\&blobtable=Mung oBlobs\&blobwhere $=1158617688340 \&$ ssbinary $=$ true

Ortega y Gasset, J. (1998). Misión de la Universidad. Madrid: Fundación Universidad-Empresa.

Ortega y Gasset (1983). Obras completas. Madrid: Revista de Occidente, Alianza, I, p. 509.

Pedro-Carañana, J. (2016). "Hacia un enfoque epistemológico pluralista en los estudios de Comunicación y Cambio: Humanismo, Ciencia y Ecologismo". Obets. Revista de Ciencias Sociales. 11 (1), p.157.

Scott, C.L. (2015). "El futuro del aprendizaje 3: ¿Qué tipo de pedagogías se necesitan para el siglo XXI?". Investigación y Prospectiva en Educación, UNESCO, 15, p.7. Disponible en: http://unesdoc.unesco.org/images/0024/002431/243126s.pdf

Todd, R. J. (2010). Curriculum integration. Learning in a changing world. Camberwell, Australia: ACER Press.

Torres Menárguez, A. (2017) "Hay que acabar con el formato de clases de 50 minutos". El País. 20 Febrero. Disponible en: https://elpais.com/economia /2017/02/17/actualidad/1487331225_284546.html?id_externo_rsoc=FB_CM 